\title{
Bioinformatic analysis of chemokine (C-C motif) ligand 21 and SPARC-like protein 1 revealing their associations with drug resistance in ovarian cancer
}

\author{
FUQIANG YIN ${ }^{1,2^{*}}$, XIA LIU $^{3 *}$, DANRONG LI ${ }^{1}$, QI WANG ${ }^{2}$, WEI ZHANG ${ }^{2}$ and $\mathrm{LI} \mathrm{LI}^{1,2}$ \\ ${ }^{1}$ Department of Gynecologic Oncology, Affiliated Tumor Hospital of Guangxi Medical University; \\ ${ }^{2}$ Medical Scientific Research Centre, ${ }^{3}$ Center for Translational Medicine, \\ Guangxi Medical University, Nanning, Guangxi 530021, P.R. China
}

Received November 20, 2012; Accepted January 14, 2013

DOI: $10.3892 /$ ijo.2013.1819

\begin{abstract}
Chemokine (C-C motif) ligand 21 (CCL21) and SPARC-like protein 1 (SPARCL1/MAST9/hevin/SC-1) are associated with various biological behavior in the development of cancers. Although the expression of CCL21 and SPARCL1 is downregulated in many solid tumors, their roles in ovarian cancer and their associations with drug resistance have rarely been studied. We performed a comprehensive bioinformatic analysis consisting of motif analysis, literature co-occurrence, gene/protein-gene/protein interaction network, protein-small molecule interaction network, and microRNAs enrichments which revealed that CCL21 and SPARCL1 directly or indirectly interact with a number of genes, proteins, small molecules and pathways associated with drug resistance in ovarian and other cancers. These results suggested that CCL21 and SPARCL1 may contribute to drug resistance in ovarian cancer. This study provided important information for further investigation of drug resistance-related functions of CCL21 and SPARCL1 in ovarian cancer.
\end{abstract}

\section{Introduction}

Ovarian cancer is the most lethal gynecologic cancer, with high rate of mortality all over the world. Early stages of ovarian cancer are generally asymptomatic and thus diagnosis usually occurs after the disease has disseminated beyond the ovaries (1). Therefore, $70 \%$ of ovarian cancer patients are diagnosed with advanced-stage disease and 5-year survival rates are less than $40 \%$, with only modestly improved survival over the past 40 year

Correspondence to: Professor Li Li, Department of Gynecologic Oncology, Affiliated Tumor Hospital of Guangxi Medical University, 71 Hedi Road, Nanning, Guangxi 530021, P.R. China

E-mail: lili@gxmu.edu.cn

*Contributed equally

Key words: bioinformatic, chemokine (C-C motif) ligand 21, SPARC-like protein 1, drug resistance, ovarian cancer
(2). Although the standard taxane/platinum regimen achieves a complete response rate of 40 to $60 \%$ in advanced ovarian cancer patients (2), relapse occurs in over $70 \%$ of the patients, resulting in drug resistance and finally leading to fatal disease (3).

Drug resistance in ovarian cancer normally develops after the treatments to advanced stage cancer patients with chemotherapies (3) and associates with aberrant expression of some genes, such as tumor suppressor genes (TSGs) and oncogenes. At least 16 candidate TSGs, 15 oncogenes, many other genes and more than 7 signaling pathways have been implicated in aberrations in cell proliferation, apoptosis, autophagy and changes in cell adhesion and motility in ovarian cancer. All of these cellular processes contribute to cancer development and metastasis (4). Among all the genes and signaling pathways participated in the development of ovarian cancer, genes [such as p53 $(5,6)$, BRCA1 (7,8), BRCA2 (8) and ERBB2 (9)] and pathways [such as p53 signaling pathway (10) and mTOR signaling pathway (11)] related to drug resistance have been identified, suggesting that genes contributing to advanced-stage ovarian cancer would also be noteworthy in drug resistance.

The latest published research of ovarian cancer reveals that CCL21 and SPARCL1 are noteworthy in ovarian cancer because their promoters are hypermethylated and silenced in the vast majority of the tumors (in a total of 489 high-grade serous ovarian adenocarcinomas), which is even more notable than $B R C A 1$ for which the promoter is hypermethylated and silenced in only 56 of 489 (11.5\%) tumors (12). BRCAl is important in the development of ovarian cancer and is involved in survival (13), metastasis (14), apoptosis (15) and drug resistance $(16,17)$. These findings suggest that CCL21 and SPARCL1 could play important roles in advanced stage ovarian cancers (12).

In this study, based on the comprehensively bioinformatics analysis through motif analysis, literature co-occurrence, protein-protein interaction network, protein-small molecule interaction network and microRNAs (miRNAs) enrichments, we found that CCL21 and SPARCL1 directly or indirectly interacted with many genes, proteins, small molecules and pathways associated with drug resistance in ovarian cancer and other cancers, suggesting that CCL21 and SPARCL1 might contribute to drug resistance in ovarian cancer. 


\section{Materials and methods}

The target genes (CCL21 and SPARCL1) silenced in the vast majority of advanced-stage ovarian adenocarcinomas (12) were selected for bioinformatics analysis.

The motif analysis of proteins was performed with SSDB Motif Search in Kyoto Encyclopedia of Genes and Genomes (KEGG) online database (http://www.genome.jp/kegg/); the pathway searches were performed with KEGG online database and GENEGO online database (http://www.genego.com/). Protein domain interactions were analyzed by DOMINE online database $(18,19)$ (http://domine.utdallas.edu/cgi-bin/Domine).

Literature Co-Occurrence was performed with Pubgene online tool (20) (http://www.pubgene.org/index.cgi); the gene/protein-gene/protein interaction network was generated with GeneMANIA (21) (http://www.genemania.org/); the protein-small molecules interaction network was generated with BiologicalNetworks 2 software (22) (downloaded from http://biologicalnetworks.net/Software/index.php).

The miRNA target-gene prediction was performed by miRWalk online tool (23) (http://www.umm.uni-heidelberg. de/apps/zmf/mirwalk/), for which 7 prediction programs (miRanda, miRDB, miRWalk, PICTAR4, PICTAR5, RNA22 and Targetscan) were selected, and the same miRNA predicted by at least 3 of these software was selected for subsequent analysis. The pathway enrichment analysis of miRNAs was performed with DIANA-mirPath web server (24) (http://diana. cslab.ece.ntua.gr/pathways/).

\section{Results}

The functions of CCL21 and SPARCL1 in cancers. CCL21 is one of the chemokines which belong to the small molecule chemoattractive cytokine family. Chemokines mediate their chemical effect on target cells through G-protein-coupled receptors, which are characterized structurally by 7 transmembrane spanning domains and involved in the attraction and activation of mononuclear and polymorphonuclear leukocytes (25). CCL21 participated in three pathways, cytokine-cytokine receptor interaction, chemokine signaling pathway and NF- $\mathrm{kB}$ signaling pathway, based on the searches in KEGG database, and participated in apoptosis and survival_Lymphotoxin- $\beta$ receptor signaling pathway based on the searches in GENEGO database. Besides, CCL21 plays roles in the regulation of ERK pathway in human non-small cell lung cancer cells (26). CCL21 is downregulated in many cancers and associated with lymph node metastasis, poor prognosis (27), apoptosis (26), cell cycle (28) and tumor growth (29). SPARCL1 belongs to SPARC family that contains ten protein members (30). SPARCL1 is widely expressed in normal and cancer tissues, and it was initially identified as an anti-adhesive extracellular matrix protein with anti-proliferative effects mediated through cell-cell adhesion $(31,32)$. In addition, SPARCL1, which is downregulated in several tumor types such as colorectal and gastric cancer, is associated with tumor diagnosis, progression and prognosis $(30,33,34)$. Therefore, SPARCL1 is considered to be a TSG (35) and may have many further unexplored functions in cancer development.

However, the studies on CCL21 and SPARCL1 associated with drug resistance are rare. Only one study reports that
SPARCL1 is an extracellular matrix remodeling gene and may contribute to drug resistance in pediatric osteosarcoma (36). The research on CCL21 and SPARCL1 in ovarian cancer is limited. It is reported that CCL21 potentiated the cytotoxicity to ovarian cancer cells (37) and SPARCL1 is inactivated in ovarian cancer (38). More recently, CCL21 and SPARCL1 are noteworthy in ovarian cancer because they are silenced in the vast majority of high-grade serous ovarian adenocarcinomas (12).

Function prediction through motif-based approaches. Conserved protein sequence motifs are short stretches of amino acid sequence patterns that potentially encode the function of proteins (39). Except in CfCCL21, IL8 domain (accession: PF00048) was a unique and highly conserved motif in human CCL21 and its homologous proteins (Table I) according to SSDB Motif Search, indicating that IL8 domain might contribute to the function of CCL21. IL8 domain originally came from IL8 protein which closely related to drug resistance in many solid tumors and cancer cells. The upregulated expression levels of IL6 and IL8 may contribute to multidrug resistance in human breast cancer cells (40). Similarly, IL8 is overexpressed in paclitaxel resistance SKOV3 cells, and therefore is considered to be associated with paclitaxel resistance (41). These studies suggested that IL8 domain might closely relate to drug resistance, indicating that CCL21 might associate with drug resistance.

Four motifs comprising FOLN domain (accession: PF09289), Kazal_1 domain (accession: PF00050), Kazal_2 domain (accession: PF07648) and SPARC_Ca_bdg region (accession: PF10591) were highly conserved in human SPARCL1 and its homologous proteins in other species (Table II), suggesting that these motifs might closely relate to the functions of SPARCL1. Besides, efhand domain (accession: PF00036) was also observed in most SPARCL1 proteins. It has been reported that serine protease inhibitor Kazal-type 1, which contains Traw_N, Kazal_1 and Kazal_2 domains, affects multiple aggressive properties in breast cancer such as survival, invasiveness, and chemoresistance (42). Similarly, serine proteinase inhibitor Kazal-type 2, which contains only Kazal_1 and Kazal_2 domains, is also found to play an important role in tumor progression and response to the treatment in leukemia cell lines (43). These studies indicated that Kazal_1 and Kazal_2 domains might be associated with drug resistance in cancers. A previous study revealed that protein phosphatase with efhand domain may correlate with stress protective responses, cell survival, growth, proliferation and drug resistance (44). S100P with efhand domain is detected in a spectrum of human tumor cell lines and tissues derived from prostate, pancreas, breast, lung and colon, in which it is connected with malignant phenotype, hormone independence and resistance to chemotherapy (45). These studies indicated that efhand domain might also associate with drug resistance in cancers. In addition, on the basis of DOMINE online analysis, FOLN domain interacted with Kazal_1 domain, SPARC_Ca_bdg region interacted with FOLN and Kazal_1 domains and efhand domain interacted with Kazal_1 domain, suggesting that all these conserved motifs of SPARCL1 were closely related and interacted with each other. Taken together, we concluded that FOLN, Kazal_1, Kazal_2, SPARC_Ca_bdg, and efhand conserved in SPARCL1 were associated with drug resistance in cancers. 
Table I. The motifs of CCL21 and its homologous proteins according to SSDB Motif Search.

\begin{tabular}{llll}
\hline & & \multicolumn{2}{c}{ Motif } \\
\cline { 2 - 4 } Protein & \multicolumn{1}{c}{ KEGG ID } & IL8 & YqzE \\
\hline HsCCL21 & hsa:6366 & $*$ & - \\
AmCCL21-like & aml:100480794 & $*$ & - \\
BtCCL21 & bta:511112 & $*$ & - \\
CfCCL21 & cfa:448796 & $*$ & $*$ \\
EcCCL21-like & ecb:100060619 & $*$ & - \\
EcCCL21-like & ecb:100063059 & $*$ & - \\
MmCCL21 & mcc:574183 & $*$ & - \\
MdCCL21-like & mdo:100028728 & $*$ & - \\
MmCCL21-like & mmu:100041504 & $*$ & - \\
MmCCL21-like & mmu:100041593 & $*$ & - \\
MmCCL21B & mmu:100042493 & $*$ & - \\
MmCCL21C-like & mmu:100862177 & $*$ & - \\
MmCCL21A & mmu:18829 & $*$ & - \\
MmCCL21C & mmu:65956 & $*$ & - \\
OaCCL21-like & oaa:100092451 & $*$ & - \\
PtCCL21 & ptr:746205 & $*$ & - \\
RnCCL21 & rno:298006 & $*$ & - \\
SsCCL21 & ssc:448797 & $*$ & - \\
XtCCL21A-like & xtr:100490971 & $*$ & - \\
\hline & & & \\
\hline
\end{tabular}

hsa, Homo sapiens; aml, Ailuropoda melanoleuca; bta, Bos Taurus; cfa, Canis familiaris; ecb, Equus caballus; mcc, Macaca mulatta; mdo, Monodelphis domestica; mmu, Mus musculus; oaa, Ornithorhynchus anatinus; ptr, Pan troglodytes; rno, Rattus norvegicus; ssc: Sus scrofa; $\mathrm{xtr}$, Xenopus tropicalis.

Function prediction and analysis based on interaction networks

Function prediction and analysis based on literature co-occurrence. The involvement of CCL21 and SPARCL1 in cancer drug resistance had not been reported on the basis of literature co-occurrence, whereas there were 10 genes co-occurring with CCL21 and SPARCL1 in ovarian cancer (Fig. 1). Among those 10 genes, p53, BRCA1 and BRCA2 are well-known TSGs, and downregulation of these 3 genes contributes to the enhancement of drug resistance in ovarian cancer (5-8). ERBB2 and BCL2 are oncogenes; ERBB2 takes part in drug resistance in ovarian cancer (9), while BCL2 is reported to participate in drug resistance in other cancers $(46,47)$. Besides, AFP is a drug resistance-related gene which plays a role in the expression of P-glycoprotein (48); TSC1 is a putative TSG participating in the signaling pathway of the mammalian target of rapamycin (mTOR) associated with proliferation, survival and drug resistance in leukemia cells (49); PTPRC, an apoptosis-related gene near cis-regulatory elements (50), is regarded as underexpression in breast cancer (51), suggesting that this gene may relate to drug resistance.

The involvement of CCL21 and SPARCL1 in ovarian cancer has rarely been studied. We observed that CCL21 had co-occurrences with p53, BCL2, PTPRC and ERBB2;
SPARCL1 had co-occurrence with p53, BCL2, PTPRC and TSC1 (Fig. 1), suggesting that they might interact directly or indirectly. Taken together, we found that 8 in 10 genes which had co-occurrences with CCL21 and SPARCL1 in 'ovarian cancer' were drug resistance-related genes in ovarian and other cancers, suggesting that CCL21 and SPARCL1 might also be involved in drug resistance.

Function prediction and analysis based on gene/proteingene/protein interactions. The functions of CCL21 and SPARCL1 were predicted using GeneMANIA (as shown in Fig. 2). CCL21 was co-expressed, co-localized, physically interacted, shared protein domains and pathways with a number of proteins, especially with CCL19, CCR7 and CCR6, suggesting that they were functionally related. In comparison, SPARCL1 had considerably fewer interactions with other proteins.

Based on the annotated functions in accordance with the GeneMANIA network (Table III), CCL21, together with other proteins, played important roles in the regulation of leukocytes, neutrophil chemotaxis, G-protein coupled receptor activity and calcium ion. It has been proven that leukocytes have close relationship with drug resistance, both in vivo and in vitro. In a 'blinded' study of 21 patients receiving combination cisplatin/carboplatin treatments, there was a direct relationship between DNA damage in leukocytes and disease response, and in leukocytes in vivo, persistence and accumulation are prominent features of the cisplatin-DNA adduct profile (52). Neutrophil chemotaxis seemed to be associated with drug resistance in an indirect way. For instance, celastrol is identified as an inhibitor of neutrophil chemotaxis, and it induces synergistic apoptosis when combined with conventional microtubule-targeting drugs and manifested efficacy toward taxol-resistant cancer cells at the cellular level (53). Similarly, stress and drug-induced interleukin-8 (IL8) signaling has been shown to confer chemotherapeutic resistance in cancer cells, while IL8 is a proinflammatory CXC chemokine contributed to the promotion of neutrophil chemotaxis and degranulation (54). G protein-coupled receptors essentially regulate all cellular processes, including those that are fundamental to cancer pathology, such as differentiation, proliferation, migration, tissue invasion, survival and drug resistance (55). Calcium content increases in multidrug resistant (MDR) cells and the resistance could be reversed by the calcium channel blocker verapamil, suggesting that calcium ion may play a role in drug resistance $(56,57)$.

There was no annotated function for SPARCL1 based on GeneMANIA, but we could deduce its function through its interactions with other proteins. As shown in Fig. 2, SPARCL1 was co-expressed with many proteins such as RAMP3 and VWF. RAMP3 is associated with receptor-mediated endocytosis which is involved in drug resistance. Hsp47/CBP2 is a favorable candidate for targeted delivery of anticancer drugs in human squamous cell carcinoma of the head and neck, and the uptake of the targeted conjugate is inhibited in the presence of an anti-Hsp47 antibody, suggesting the involvement of active receptor mediated endocytosis in cell entry of the conjugate (58). The VWF is related to cell-substrate adhesion which is also involved in drug resistance. It has been proven that cell-substrate adhesion contributes to drug resistance via apoptosis in acute myeloid leukaemia, small cell lung cancer cells, breast cancer and glioblastoma cells (59). 


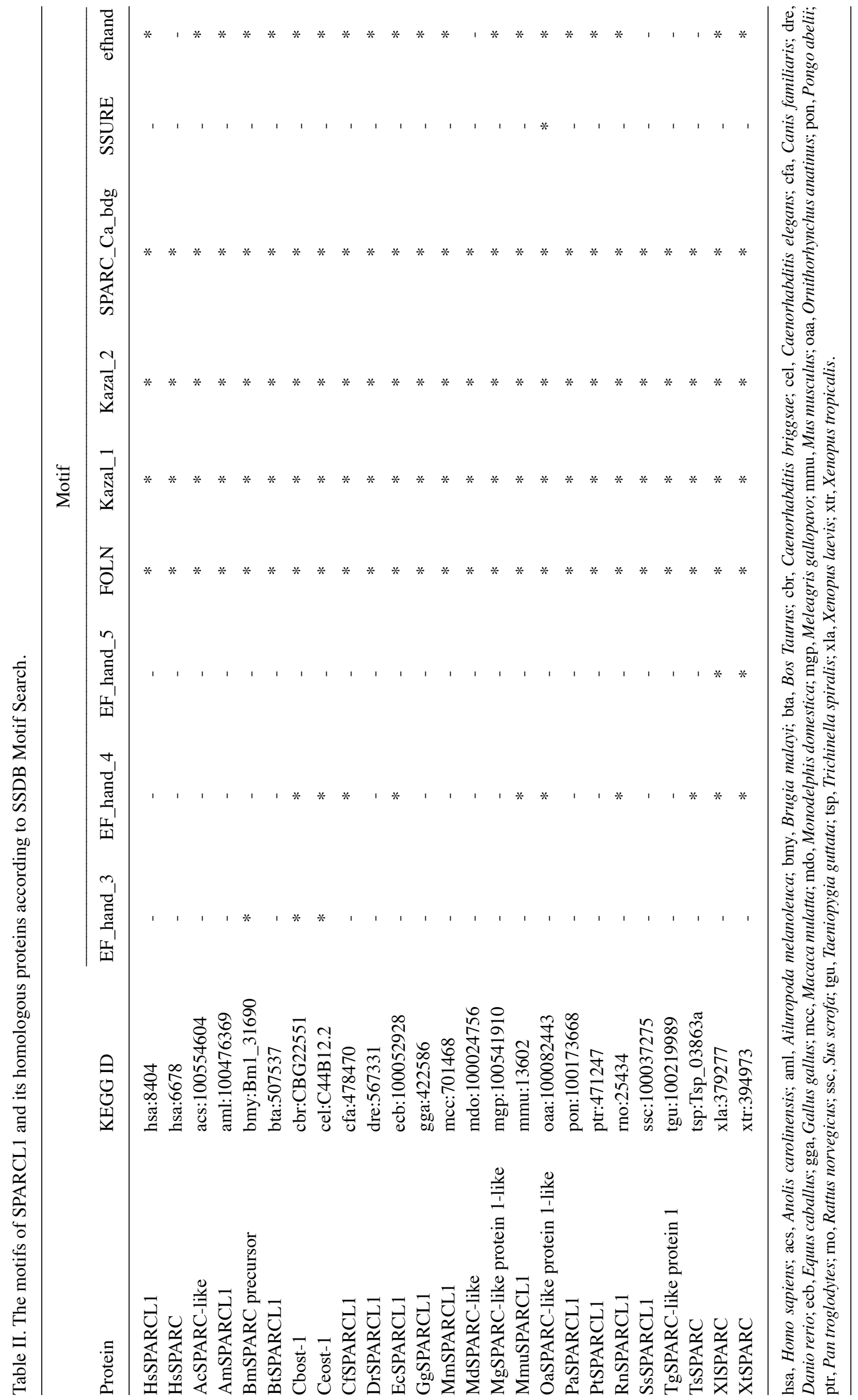




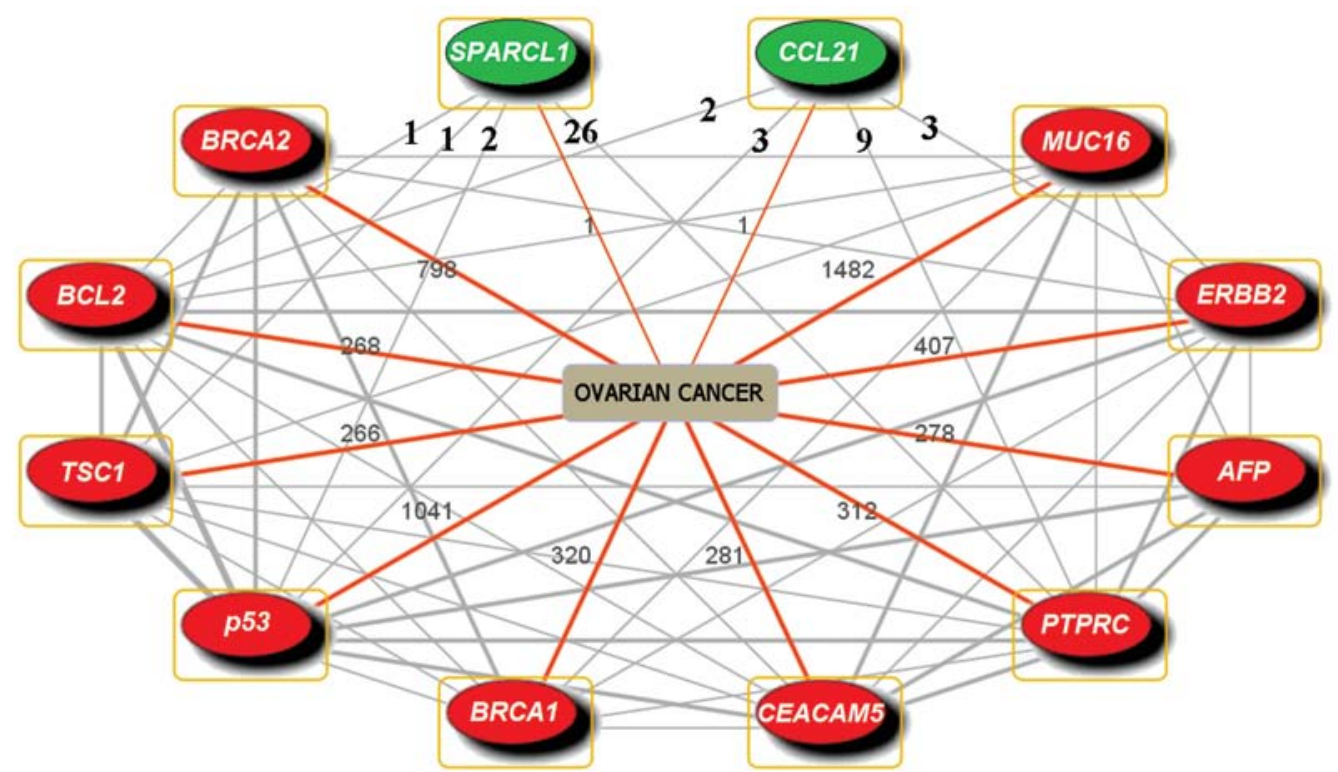

Figure 1. The co-occurrence analysis of CCL21 and SPARCL1 to the exact keyword expression 'ovarian cancer' by Pubgene online database and tool. The orange line indicates the co-occurrence of the genes to the key word 'ovarian cancer', and the number beside the line is the number of co-occurrence between the gene and 'ovarian cancer'; the gray line indicates the co-occurrence of the genes between each other, the number on the gray line is the literature number for CCL21 and SPARCL1 with their co-occurring genes.

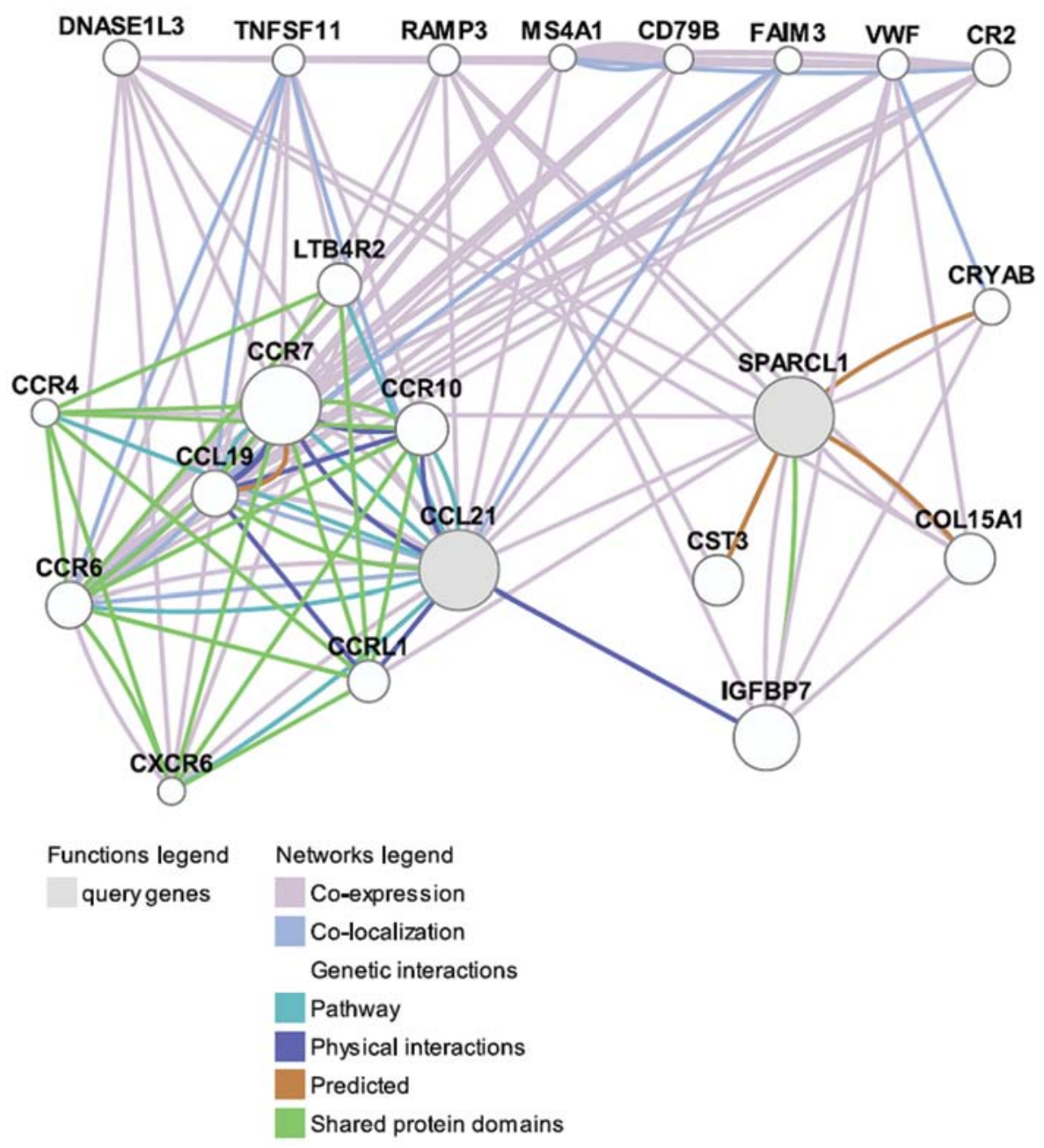

Figure 2. Function prediction of CCL21 and SPARCL1 using GeneMANIA. The type of interactions between genes/proteins was illustrated as the network legend indicates. 
Table III. The annotated functions of CCL21 and other proteins related to drug resistance in GeneMANIA network (as shown in Fig. 2).

\begin{tabular}{lcc} 
GO annotation & FDR (n/a) ${ }^{\mathrm{a}}$ & Genes/proteins in the network \\
\hline $\begin{array}{l}\text { Regulation of leukocyte chemotaxis, } \\
\text { apoptosis, migration and activation }\end{array}$ & $6.22 \mathrm{E}-05$ to 1.44E-02 & CCL21, CCL19, CCR7, CCR6, TNFSF11 \\
$\begin{array}{l}\text { Regulation of neutrophil chemotaxis } \\
\text { G-protein coupled receptor activity }\end{array}$ & $2.92 \mathrm{E}-04$ to 1.44E-03 & CCL21, CCL19, CCR7 \\
Calcium ion concentration, homeostasis, & $3.18 \mathrm{E}-05$ to 9.39E-03 & CCR7, CCR6, CCRL1, CCR4 \\
transportation and sequestering & & \\
Positive regulation of cell adhesion & $1.57 \mathrm{E}-04$ to 6.21E-02 & CCL21, CCL19, CCR7, CCR6, CCR4 \\
Receptor-mediated endocytosis & $7.01 \mathrm{E}-03$ & CCL21, CCR7, TNFSF11 \\
Cell-substrate adhesion & $1.80 \mathrm{E}-02$ & CCL21, CCL19, RAMP3 \\
\hline
\end{tabular}

${ }^{\mathrm{a} F D R}$, false discovery rate.

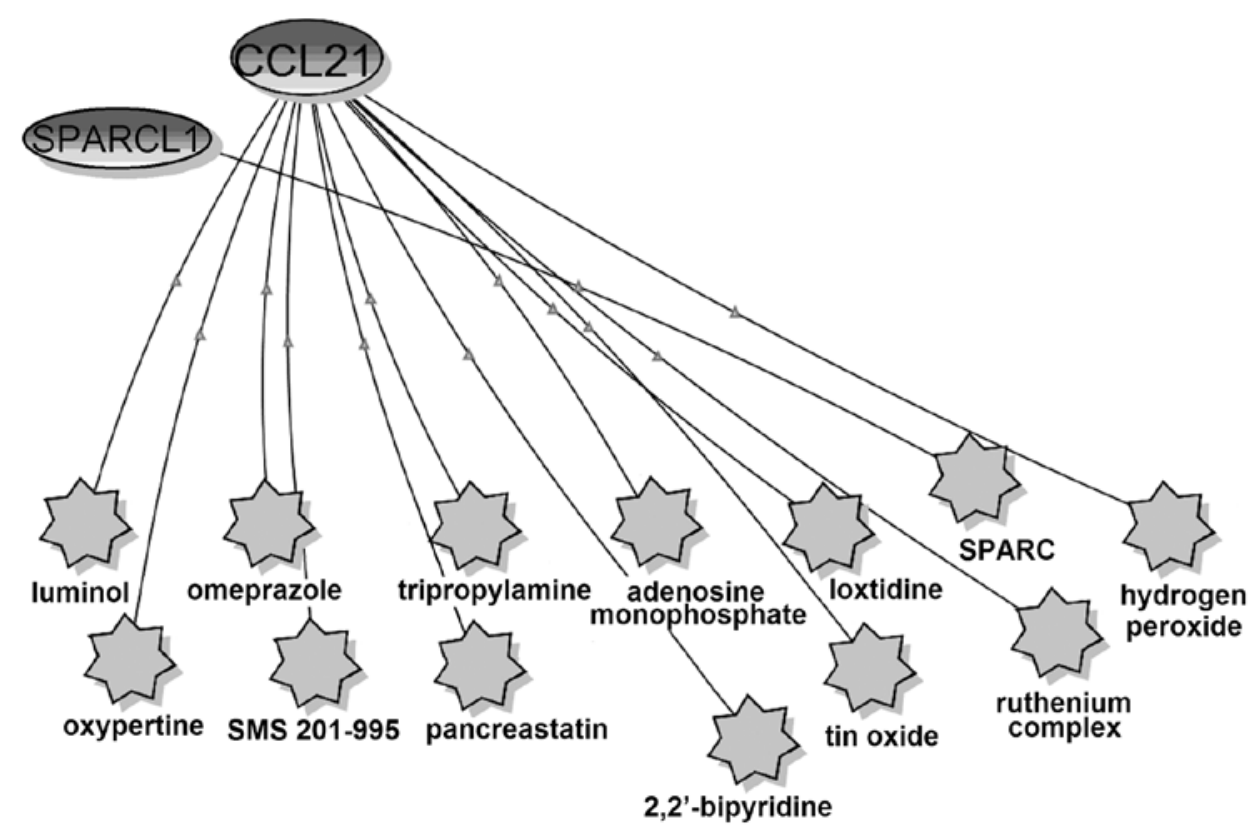

Figure 3. The interaction networks of CCL21 and SPARCL1 with small molecules by BiologicalNetworks. All the interactions between protein and small molecule were co-citations.

In addition, CCL21 and SPARCL1 were co-expressed and interacted with each other indirectly through interacting with other proteins. IGFBP7 was found to be the most important one for CCL21 and SPARCL1 interactions. IGFBP7 had very strong physical interactions with CCL21 and shared protein domains with SPARCL1, indicating that CCL21, IGFBP7 and SPARCL1 might be functionally related. IGFBP7 has been identified as one of these factors responsible for the establishment and/or maintenance of oncogene-induced senescence, and has been shown to be a TSG in a variety of solid cancers (60). Aberrant expression of IGFBP7 in adult leukemia is correlated with chemotherapy resistance and shorter survival. Addition of IGFBP7 to leukemic cell lines inhibits cell growth without induction of apoptosis or senescence, suggesting a role of IGFBP7 in contributing to drug resistance through reduced sensitivity to cytostatic drugs (61).
Function prediction and analysis based on protein-small molecules interactions. The relationship of CCL21, SPARCL1 and small molecules were analyzed using BiologicalNetworks (Fig. 3). SPARCL1 had co-citation with only one small molecule, SPARC, which is the peptides of SPARC protein SPARC $_{113-130}$ and $\mathrm{SPARC}_{54-73}$ ) (62). SPARCL1 exhibits $62 \%$ identity with the anti-adhesive extracellular matrix protein SPARC, over a region of 232 aa spanning more than four-fifths of the SPARC coding sequence (63). SPARCL1 shared four domains (FOLN, Kazal_1, Kazal_2 and SPARC_Ca_bdg) with SPARC based on the SSDB Motif Search results (Table II). Thus, SPARCL1 might have similar functions with SPARC. SPARC is a candidate TSG and a putative resistance-reversal gene and plays an important part in drug resistance in ovarian cancer $(64,65)$. Therefore, we concluded that SPARCL1, which is considered as a TSG (35), might also contribute to drug resistance in ovarian cancer. 
CCL21 had co-citations with 12 small molecules, and half of them comprising omeprazole, SMS 201-995, adenosine monophosphate, ruthenium complex, hydrogen peroxide and 2,2'-bipyridine are associated with drug resistance in cancers. In vivo experiments show that oral pretreatment with omeprazole induces a sensitivity of human solid tumors to anticancer drugs (66). SMS 201-995 is proven to stimulate prostatic tumor growth and may sensitize tumor cells to subsequent chemotherapy (67). Adenosine monophosphate may participate in drug resistance of ovarian cancer through adenosine monophosphateactivated protein kinase pathway (68). The ruthenium complexes are effective tumor-inhibiting drugs in experimental therapy of autochthonous colorectal carcinomas in rats, and they can be promising candidate drugs in the second-line treatment of colorectal cancers resistant to other cytostatic drugs (69). Thioredoxin has much higher levels in all cisplatin-resistant human bladder and prostatic cancer cell lines compared with their drug-sensitive parental counterpart, and downregulation of its expression can increase cell sensitivity to cisplatin and also to other superoxide-generating agents including hydrogen peroxide, suggesting that hydrogen peroxide may also relate to drug resistance (70); 2,2'-bipyridine is a well-characterized chelating agent known to have anti-proliferative activity that links to drug resistance (71).

Function prediction and analysis based on KEGG pathways modulated by miRNAs. Total of 37 and 31 miRNAs were predicted to be the transcriptional targets of CCL21 and SPARCL1 through miRWalk, respectively. The pathway enrichment analysis of those miRNAs was performed with DIANA-mirPath, and an overview of the parts of the pathway modulated by miRNAs was integrated. Among all the pathways modulated by miRNAs targeted CCL21 and SPARCL1, 11 of them are involved in drug resistance in ovarian and many other cancers (Table IV).

Van Jaarsveld et al (72) systematically reviewed the miRNAs related to drug resistance in ovarian cancer. Among the miRNAs, some were the transcriptional targets of CCL21 and SAPRCL1 (Table IV). For instance, hsa-miR-125b and hsa-miR-370 were the targets of CCL21. Hsa-miR-125b is downregulated in paclitaxel resistant A2780 cell lines, therefore suggesting a direct involvement in the development of chemoresistance (73). Hsa-miR-370 is upregulated in platinum resistant EOC (74), suggesting that hsa-miR-370 may contribute to drug resistance through downregulation its target genes. Similarly, hsa-miR-431, the transcriptional target of SPARCL1, is also upregulated in topotecan resistant ovarian cancer cells (75). The pathway enrichment analysis of miRNAs related to drug resistance in ovarian cancer has also been studied. For example, it has been reported that $11 \mathrm{miRNAs}$ are differentially expressed in cisplatin resistant ovarian cancer cells, which potentially target many important pathways comprising MAPK, Wnt signaling, mTOR signaling, apoptosis and many other signaling pathways which are all related to drug resistance in cancers (76).

All of these 11 drug resistance-related pathways modulated by the miRNAs targeted CCL21 and SPARCL1 (Table IV) were proven to be involved in drug resistance in ovarian cancer. The Wnt signaling pathway participates in drug resistance through inducing apoptosis and inhibiting tumor growth $(77,78)$. Cell adhesion molecule is overexpressed in ovarian cancer, espe- cially in recurrent/chemotherapy-resistant epithelial ovarian cancer, suggesting that cell adhesion molecule and its pathway may play a role in drug resistance (79). p53 signaling pathway is a well-studied and contributes to the whole process of cancer developments and it is involved in drug resistance in ovarian cancer through regulating cell proliferation following DNA damage (10). Cell communication is important to tumor mechanisms and relevant to the acquisition of drug resistance in ovarian cancer (80). With the better understanding of the relationship between cell cycle and the impact of chemotherapeutic agents on the cell cycle, it becomes apparent that this physiology can create drug resistance, therefore reducing combination chemotherapeutic efficacy $(81,82)$. Amplified PI3K and activated Akt have been observed in $12-68 \%$ of tumors, and are closely associated with upregulation of mTOR signaling (83), therefore, activation of the PI3K/Akt pathway and its downstream mTOR signaling appear to represent drug resistance and poor prognosis $(11,83)$; apoptosis plays an important role in the maintenance of physiological homeostasis in response to stimuli. When the apoptosis machinery fails, abnormal cells can survive, resulting in unopposed tissue growth and eventually fatal disease such as cancer (84). Apoptosis has been demonstrated to be involved in drug resistance in many solid tumors including ovarian cancer $(85,86)$. VEGF signaling pathway is a key pathway in normal ovarian physiology and ovarian cancer, and closely related to drug resistance (87). Autophagy is involved in nucleus accumbens-1 mediated resistance to cisplatin, which is known to have important roles in proliferation, growth of tumor cells and chemotherapy resistance. Thus, the regulation of autophagy is considered to be involved in drug resistance in ovarian cancer (88). ABC transporters participated in drug resistance through controlling the drug transportation $(89,90)$.

Taken together, we found that all the 11 pathways modulated by the miRNAs targeted CCL21 and SPARCL1 contributing to drug resistance in ovarian cancer, suggesting that CCL21 and SPARCL1 may also be involved in drug resistance in ovarian cancer.

\section{Discussion}

Drug resistance, comprising both intrinsic and acquired resistance, is believed to cause treatment failure in over $90 \%$ of patients with metastatic cancer (102). Apparently, the survival of cancer patients would be highly increased if drug resistance could be overcome. There are many factors affecting drug sensitivity and cancer cell resistance to chemotherapy can occur at many levels, including drug transportation, drug inactivation, alterations in drug target, processing of drug-induced damage and failure of apoptosis (102). In ovarian cancer, some mechanisms on drug resistance have been revealed. A decrease in cell-associated drug, altered GSH-mediated metabolism and enhanced DNA repair may play roles in cellular resistance to cisplatin and alkylating agents (103). Further studies show that the increased anti-apoptotic regulator activity, increased DNA repair activity, defective DNA damage response, deregulation of growth factor receptor and post-translational modification or altered expression of $\beta$-tubulin and other microtubule regulatory proteins may be involved in drug resistance in ovarian cancer (73). Among all these mechanisms and factors which contribute to drug resistance, some are essentially involved in 


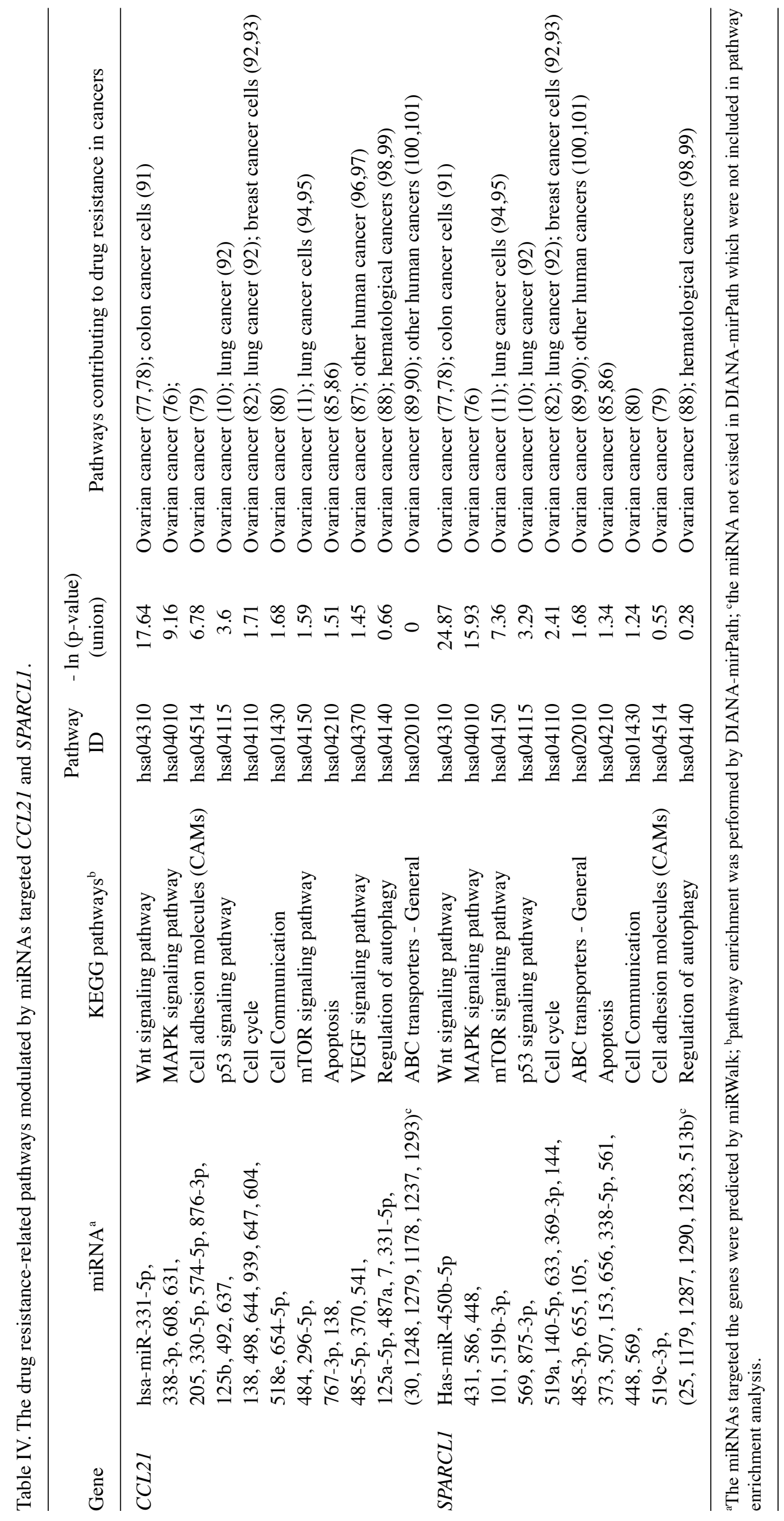




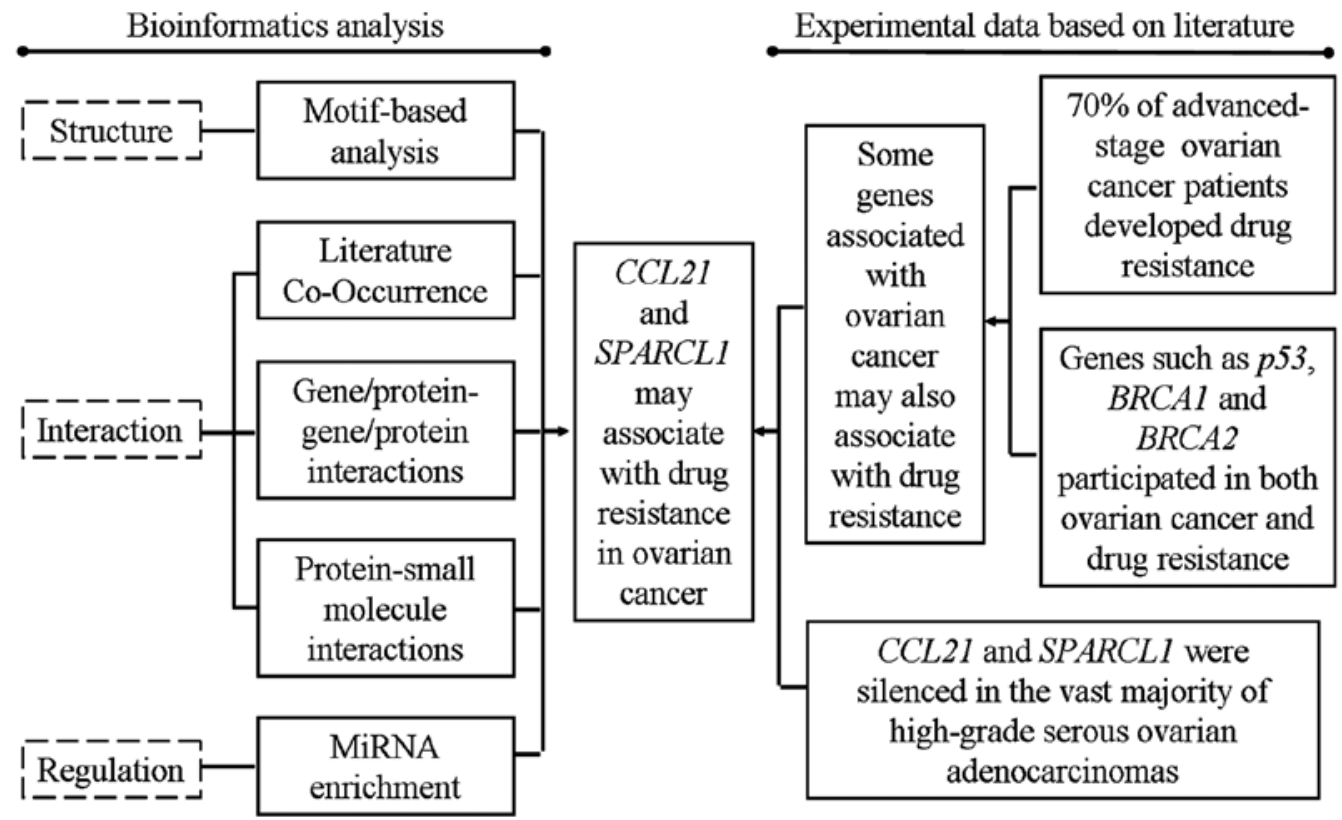

Figure 4. The overall procedure of bioinformatics analysis of CCL21 and SPARCL1 associated with drug resistance in ovarian cancer.

aberrant expression of genes. Thus, mining and exploring of potentially drug resistance-related genes would be a feasible and reasonable way to solve the drug resistance in ovarian cancer.

Gene function prediction based on bioinformatics analysis is a potential, feasible and valuable way for gene function mining, and many large-scale networks of molecular interactions within the cell have made it possible to go beyond one dimensional approaches to study protein function in the context of a network (104). Pubgene is a gene/protein database and web-based tool for literature mining. It carries out automated extraction of experimental and theoretical biomedical knowledge from publicly available gene and text databases to create a gene-to-gene co-citation network for 13,712 named human genes by automated analysis of titles and abstracts in over 10 million MEDLINE records (20). Therefore, gene and protein names are cross-referenced to each other and to terms that are relevant to understanding their biological function and importance in disease. GeneMANIA is a web-based database and tool for prediction of genes function on the basis of multiple networks derived from different genomic or proteomic data/ sources. It is fast enough to predict gene function with great accuracy (21). BiologicalNetworks server allows easy retrieval, construction and visualization of complex biological networks, including genome-scale integrated networks of protein-DNA, protein-protein and genetic interactions. Most importantly, BiologicalNetworks satisfy the need for analysis of expression profiles of genes or proteins simultaneously on to small molecules (metabolic) and cellular networks (22). Thus, the predicted functions of CCL21 and SPARCL1 based on these networks were reasonable and reliable.

Based on the network analyses, we found that 8 of the 10 genes which co-occurred with CCL21 and SPARCL1 in ovarian cancer were drug resistance-related genes (Fig. 1). Among these genes, p53 (5,6), BRCA1 (7,8), BRCA2 (8), and ERBB2 (9) are already proven to be involved in drug resistance in ovarian cancer. CCL21 and SPARCL1 were co-expressed, co-localized, physically interacted and shared protein domains and pathways with other genes/proteins according to GeneMANIA network (Fig. 2). Annotated functions (Table III) suggested that CCL21 might participate in drug resistance through regulation of leukocytes, neutrophil chemotaxis, G-protein coupled receptor activity and calcium ion, therefore, CCL21 might be a potential drug resistance-related gene. Even though SPARCL1 had no annotated functions, it was co-expressed with RAMP3 and VWF, and shared protein domains with IGFBP7 (Fig. 2), which are all reported to be associated with drug resistance $(58,59,61)$. SPARCL1 was co-expressed with CCL21, and interacted with each other through other genes, indicating that SPARCL1 might have a close relationship with CCL21 in functions. In addition, SPARCL1 exhibits $62 \%$ identity (63) and shares four domains (FOLN, Kazal_1, Kazal_2 and SPARC_Ca_bdg) with SPARC (Table II), which plays an important part in drug resistance in ovarian cancer $(64,65)$. CCL21 had co-citations with 12 small molecules according to BiologicalNetworks (Fig. 3). Among them, omeprazole, SMS 201-995, ruthenium complex, hydrogen peroxide and 2,2'-bipyridine which are demonstrated to be related to drug resistance in cancers $(66,67,69-71)$ and adenosine monophosphate is associated with drug resistance in ovarian cancer (68). Among all the genes, proteins and small molecules which had interactions with CCL21 and SPARCL1, most of them are participating in drug resistance of cancers, and some of them contribute to drug resistance in ovarian cancer. Therefore, we concluded that both CCL21 and SPARCL1 might have close relationships with drug resistance in ovarian cancer.

MicroRNAs (miRNAs) are a class of small (22 bp) endogenous non-coding RNAs which regulate gene expression mainly by its binding to the 3'-UTR of the target mRNA, and causing mRNA cleavage, destabilization or translational 
repression $(105,106)$. miRNA-mediated post-transcriptional gene regulation is considered as a significant regulator of many cellular processes, both physiological and pathological $(107,108)$. It has been proven that miRNAs play important roles in drug resistance of many cancers including ovarian cancer (73). Because miRNAs perform their functions through the regulation on their target genes, and it has been well established that miRNAs represent a class of genes with a great potential for use in diagnostics, prognosis and therapy (109), therefore, we can predict the gene function through the functions of miRNAs targeting the gene.

MiRWalk is a comprehensive database on miRNAs, which gathers predicted and validated miRNA binding sites on all mRNAs, mitochondrial genes and $10 \mathrm{~kb}$ upstream flanking regions of all known genes of human, mouse and rat. More importantly, the miRWalk is a real-time database to some extent, in which the 'Validated Target module' is updated every month and the 'Predicted Target module' is updated every 6 months (23). DIANA-mirPath is a web-based computational tool developed to identify molecular pathways potentially modulated by the expression of miRNAs. The software performs an enrichment analysis of multiple miRNA target genes comparing each set of miRNA targets to all known KEGG pathways. The output of the program shows an overview of the parts of the pathway modulated by miRNAs, facilitating the interpretation and presentation of the results of the analysis and genes (24).

Based on the analysis of miRWalk and DIANA-mirPath, we found that among all the pathways enriched by multiple miRNAs targeted CCL21 and SPARCL1, there were 11 pathways (Table IV) closely associated with drug resistance in ovarian cancer, indicating that CCL21 and SPARCL1 might contribute to drug resistance through those miRNAs to modulate drug resistance-related pathways.

Collectively, based on the function prediction using motifbased approaches, network interactions, pathway enrichment analysis of miRNAs and function predictions on the basis of experimental data from literature (Fig. 4), we concluded that CCL21 and SPARCL1 might contribute to drug resistance in ovarian cancer. This is the first report of the drug resistance-functions of CCL21 and SPARCL1 in ovarian cancer, and thus this study might set the stage for further experimental studies of CCL21 and SPARCL1 with their drug resistance associations in ovarian cancer. This study provided important information for further investigation of drug resistance-related functions of CCL21 and SPARCL1 in ovarian cancer.

\section{Acknowledgements}

We thank Keqiang $\mathrm{Wu}$ at National Taiwan University for revising the manuscript.

\section{References}

1. Balch C, Huang TH, Brown R and Nephew KP: The epigenetics of ovarian cancer drug resistance and resensitization. Am J Obstet Gynecol 191: 1552-1572, 2004.

2. Jemal A, Siegel R, Ward E, Hao Y, Xu J, Murray T and Thun MJ: Cancer statistics, 2008. CA Cancer J Clin 58: 71-96, 2008.

3. Cannistra SA: Cancer of the ovary. N Engl J Med 351: 2519-2529, 2004.

4. Bast RC Jr, Hennessy B and Mills GB: The biology of ovarian cancer: new opportunities for translation. Nat Rev Cancer 9: 415-428, 2009.
5. Fraser M, Bai T and Tsang BK: Akt promotes cisplatin resistance in human ovarian cancer cells through inhibition of p53 phosphorylation and nuclear function. Int J Cancer 122: 534-546, 2008

6. Hagopian GS, Mills GB, Khokhar AR, Bast RC Jr and Siddik ZH: Expression of p53 in cisplatin-resistant ovarian cancer cell lines: modulation with the novel platinum analogue (1R, 2R-diaminocyclohexane)(trans-diacetato)(dichloro)platinum(IV). Clin Cancer Res 5: 655-663, 1999.

7. Zhou C, Smith JL and Liu J: Role of BRCA1 in cellular resistance to paclitaxel and ionizing radiation in an ovarian cancer cell line carrying a defective BRCA1. Oncogene 22: 2396-2404, 2003.

8. Yang D, Khan S, Sun Y, Hess K, Shmulevich I, Sood AK and Zhang W: Association of BRCA1 and BRCA2 mutations with survival, chemotherapy sensitivity, and gene mutator phenotype in patients with ovarian cancer. JAMA 306: 1557-1565, 2011.

9. Wu L, Wu A and Jiang K: Effect of antisense c-erbB2 on biologic behaviour and chemotherapeutic drug sensitivity in human ovarian cancer cells. Zhonghua Fu Chan Ke Za Zhi 31: 169-172, 1996 (In Chinese).

10. Benoit DS, Henry SM, Shubin AD, Hoffman AS and Stayton PS: pH-responsive polymeric sirna carriers sensitize multidrug resistant ovarian cancer cells to doxorubicin via knockdown of polo-like kinase 1. Mol Pharm 7: 442-455, 2010.

11. Itamochi $\mathrm{H}$ : Targeted therapies in epithelial ovarian cancer: molecular mechanisms of action. World J Biol Chem 1: 209-220, 2010.

12. Cancer Genome Atlas Research Network: Integrated genomic analyses of ovarian carcinoma. Nature 474: 609-615, 2011.

13. Narod S, Moody J, Rosen B, Fan I, Risch A, Sun P and McLaughlin J: Estimating survival rates after ovarian cancer among women tested for BRCA1 and BRCA2 mutations. Clin Genet: June 8, 2012 (Epub ahead of print).

14. Szabova L, Yin C, Bupp S, et al: Perturbation of Rb, p53, and Brcal or Brca 2 cooperate in inducing metastatic serous epithelial ovarian cancer. Cancer Res 72: 1-13, 2012.

15. Thangaraju M, Kaufmann SH and Couch FJ: BRCA1 facilitates stress-induced apoptosis in breast and ovarian cancer cell lines. J Biol Chem 275: 33487-33496, 2000.

16. Connor JP, Felder M, Kapur A and Onujiogu N: DcR3 binds to ovarian cancer via heparan sulfate proteoglycans and modulates tumor cells response to platinum with corresponding alteration in the expression of BRCA1. BMC Cancer 12: 176, 2012.

17. Quinn JE, James CR, Stewart GE, et al: BRCA1 mRNA expression levels predict for overall survival in ovarian cancer after chemotherapy. Clin Cancer Res 13: 7413-7420, 2007.

18. Yellaboina S, Tasneem A, Zaykin DV, Raghavachari B and Jothi R: DOMINE: a comprehensive collection of known and predicted domain-domain interactions. Nucleic Acids Res 39: D730-D735, 2011.

19. Raghavachari B, Tasneem A, Przytycka TM and Jothi R: DOMINE: a database of protein domain interactions. Nucleic Acids Res 36: D656-D661, 2008.

20. Jenssen TK, Laegreid A, Komorowski J and Hovig E: A literature network of human genes for high-throughput analysis of gene expression. Nat Genet 28: 21-28, 2001.

21. Mostafavi S, Ray D, Warde-Farley D, Grouios C and Morris Q: GeneMANIA: a real-time multiple association network integration algorithm for predicting gene function. Genome Biol 9 (Suppl 1): S4, 2008.

22. Baitaluk M, Sedova M, Ray A and Gupta A: BiologicalNetworks: visualization and analysis tool for systems biology. Nucleic Acids Res 34: W466-W471, 2006.

23. Dweep H, Sticht C, Pandey P and Gretz N: miRWalk - database: prediction of possible miRNA binding sites by 'walking' the genes of three genomes. J Biomed Inform 44: 839-847, 2011.

24. Papadopoulos GL, Alexiou P, Maragkakis M, Reczko M and Hatzigeorgiou AG: DIANA-mirPath: integrating human and mouse microRNAs in pathways. Bioinformatics 25: 1991-1993, 2009.

25. Baggiolini M, Dewald B and Moser B: Human chemokines: an update. Annu Rev Immunol 15: 675-705, 1997.

26. Xu Y, Liu L, Qiu X, et al: CCL21/CCR7 prevents apoptosis via the ERK pathway in human non-small cell lung cancer cells. PLoS One 7: e33262, 2012.

27. Hwang TL, Lee LY, Wang CC, Liang Y, Huang SF and Wu CM: CCL7 and CCL21 overexpression in gastric cancer is associated with lymph node metastasis and poor prognosis. World $\mathbf{J}$ Gastroenterol 18: 1249-1256, 2012. 
28. Xu Y, Liu L, Qiu X, et al: CCL21/CCR7 promotes G2/M phase progression via the ERK pathway in human non-small cell lung cancer cells. PLoS One 6: e21119, 2011.

29. Yousefieh N, Hahto SM, Stephens AL and Ciavarra RP Regulated expression of CCL21 in the prostate tumor microenvironment inhibits tumor growth and metastasis in an orthotopic model of prostate cancer. Cancer Microenviron 2: 59-67, 2009.

30. Zhang H, Widegren E, Wang DW and Sun XF: SPARCL1: a potential molecule associated with tumor diagnosis, progression and prognosis of colorectal cancer. Tumour Biol 32 1225-1231, 2011

31. Hambrock HO, Nitsche DP, Hansen U, Bruckner P, Paulsson M, Maurer $\mathrm{P}$ and Hartmann U: SC1/hevin. An extracellular calcium-modulated protein that binds collagen I. J Biol Chem 278: 11351-11358, 2003

32. Girard JP and Springer TA: Modulation of endothelial cell adhesion by hevin, an acidic protein associated with high endothelial venules. J Biol Chem 271: 4511-4517, 1996.

33. Li P, Qian J, Yu G, Chen Y, Liu K, Li J and Wang J: Down-regulated SPARCL1 is associated with clinical significance in human gastric cancer. J Surg Oncol 105: 31-37, 2012.

34. Yu SJ, Yu JK, Ge WT, Hu HG, Yuan Y and Zheng S: SPARCL1, Shp2, MSH2, E-cadherin, p53, ADCY-2 and MAPK are prognosis-related in colorectal cancer. World J Gastroenterol 17: 2028-2036, 2011.

35. Sullivan MM and Sage EH: Hevin/SC1, a matricellular glycoprotein and potential tumor-suppressor of the SPARC/BM-40/ Osteonectin family. Int J Biochem Cell Biol 36: 991-996, 2004.

36. Mintz MB, Sowers R, Brown KM, et al: An expression signature classifies chemotherapy-resistant pediatric osteosarcoma. Cancer Res 65: 1748-1754, 2005.

37. Song J, Wang X, Lei C, et al: Fusion of chemotactic peptide to a single-chain bi-specific antibody ( $\mathrm{scBsAb}$ ) potentiates its cytotoxicity to target tumour cells. Biotechnol Appl Biochem 45: 147-154, 2006

38. Biade S, Marinucci M, Schick J, et al: Gene expression profiling of human ovarian tumours. Br J Cancer 95: 1092-1100, 2006.

39. Lu X, Zhai C, Gopalakrishnan V and Buchanan BG: Automatic annotation of protein motif function with Gene Ontology terms BMC Bioinformatics 5: 122, 2004

40. Yang W, Chen LP, Huang R and Huang RP: Inhibition of IL-6 and IL-8 enhances chemosensitization in multidrug resistant human breast cancer cells. AACR Meeting Abstracts 2005 : $1199-c, 2005$

41. Duan Z, Feller AJ, Penson RT, Chabner BA and Seiden MV: Discovery of differentially expressed genes associated with paclitaxel resistance using cDNA array technology: analysis of interleukin (IL) 6 , IL-8, and monocyte chemotactic protein in the paclitaxel-resistant phenotype. Clin Cancer Res 5: 3445-3453, 1999.

42. Soon WW, Miller LD, Black MA, et al: Combined genomic and phenotype screening reveals secretory factor SPINK1 as an invasion and survival factor associated with patient prognosis in breast cancer. EMBO Mol Med 3: 451-464, 2011.

43. Chen T, Lee TR, Liang WG, Chang WS and Lyu PC Identification of trypsin-inhibitory site and structure determination of human SPINK2 serine proteinase inhibitor. Proteins 77: 209-219, 2009.

44. Kutuzov MA, Bennett N and Andreeva AV: Protein phosphatase with EF-hand domains 2 (PPEF2) is a potent negative regulato of apoptosis signal regulating kinase-1 (ASK1). Int J Biochem Cell Biol 42: 1816-1822, 2010.

45. Gibadulinova A, Tothova V, Pastorek J and Pastorekova S: Transcriptional regulation and functional implication of S100P in cancer. Amino Acids 41: 885-892, 2011.

46. Zhu W, Xu H, Zhu D, et al: $\mathrm{miR}-200 \mathrm{bc} / 429$ cluster modulates multidrug resistance of human cancer cell lines by targeting BCL2 and XIAP. Cancer Chemother Pharmacol 69: 723-731, 2012.

47. Hong JH, Lee E, Hong J, Shin YJ and Ahn H: Antisense Bcl2 oligonucleotide in cisplatin-resistant bladder cancer cell lines. BJU Int 90: 113-117, 2002.

48. Dhar DK, Nagasue N, Yoshimura H, et al: Overexpression of P-glycoprotein in untreated AFP-producing gastric carcinoma. J Surg Oncol 60: 50-54, 1995.

49. Xu Z, Wang M, Wang L, Wang Y, Zhao X, Rao Q and Wang J: Aberrant expression of TSC2 gene in the newly diagnosed acute leukemia. Leuk Res 33: 891-897, 2009.

50. Liu Z and Chen S: ER regulates an evolutionarily conserved apoptosis pathway. Biochem Biophys Res Commun 400: 34-38, 2010 .
51. Perou CM, Sorlie T, Eisen MB, et al: Molecular portraits of human breast tumours. Nature 406: 747-752, 2000.

52. Dabholkar M, Bradshaw L, Parker RJ, Gill I, Bostick-Bruton F, Muggia FM and Reed E: Cisplatin-DNA damage and repair in peripheral blood leukocytes in vivo and in vitro. Environ Health Perspect 98: 53-59, 1992.

53. Jo H, Loison F, Hattori H, Silberstein LE, Yu H and Luo HR: Natural product Celastrol destabilizes tubulin heterodimer and facilitates mitotic cell death triggered by microtubule-targeting anti-cancer drugs. PLoS One 5: e10318, 2010.

54. Waugh DJ and Wilson C: The interleukin-8 pathway in cancer. Clin Cancer Res 14: 6735-6741, 2008.

55. Herr DR: Potential use of G protein-coupled receptor-blocking monoclonal antibodies as therapeutic agents for cancers. Int Rev Cell Mol Biol 297: 45-81, 2012.

56. Ma Q, Zhang ZS, Zhang YL and Lai ZS: Relationship between multidrug resistance in human colon carcinoma LoVo/Adr cell line and intracellular calcium ion concentration. Ai Zheng 21: 846-849, 2002 (In Chinese).

57. Liang $X$ and Huang Y: Intracellular free calcium concentration and cisplatin resistance in human lung adenocarcinoma A549 cells. Biosci Rep 20: 129-138, 2000.

58. Nan A, Ghandehari H, Hebert C, Siavash H, Nikitakis N, Reynolds M and Sauk JJ: Water-soluble polymers for targeted drug delivery to human squamous carcinoma of head and neck. J Drug Target 13: 189-197, 2005.

59. Westhoff MA and Fulda S: Adhesion-mediated apoptosis resistance in cancer. Drug Resist Updat 12: 127-136, 2009.

60. Benatar T, Amemiya Y, Yang W and Seth A: Insulin-likegrowth factor-binding-protein 7: an antagonist to breast cancer. In: Breast Cancer - Focusing Tumor Microenvironment, Stem cells and Metastasis. Gunduz M (ed). InTech, Rijeka, pp39-68, 2011.

61. Heesch S, Schlee C, Neumann M, et al: BAALC-associated gene expression profiles define IGFBP7 as a novel molecular marker in acute leukemia. Leukemia 24: 1429-1436, 2010.

62. Lane TF, Iruela-Arispe ML, Johnson RS and Sage EH: SPARC is a source of copper-binding peptides that stimulate angiogenesis. J Cell Biol 125: 929-943, 1994.

63. Girard JP and Springer TA: Cloning from purified high endothelial venule cells of hevin, a close relative of the antiadhesive extracellular matrix protein SPARC. Immunity 2: 113-123, 1995.

64. Socha MJ, Said N, Dai Y, et al: Aberrant promoter methylation of SPARC in ovarian cancer. Neoplasia 11: 126-135, 2009.

65. Tai IT, Dai M, Owen DA and Chen LB: Genome-wide expression analysis of therapy-resistant tumors reveals SPARC as a novel target for cancer therapy. J Clin Invest 115: 1492-1502, 2005.

66. De Milito A and Fais S: Proton pump inhibitors may reduce tumour resistance. Expert Opin Pharmacother 6: 1049-1054, 2005.

67. Logothetis CJ, Hossan EA and Smith TL: SMS 201-995 in the treatment of refractory prostatic carcinoma. Anticancer Res 14: 2731-2734, 1994.

68. Matrone A, Grossi V, Chiacchiera F, et al: p38alpha is required for ovarian cancer cell metabolism and survival. Int J Gynecol Cancer 20: 203-211, 2010.

69. Kapitza S, Pongratz M, Jakupec MA, et al: Heterocyclic complexes of ruthenium (III) induce apoptosis in colorectal carcinoma cells. J Cancer Res Clin Oncol 131: 101-110, 2005.

70. Yokomizo A, Ono M, Nanri H, et al: Cellular levels of thioredoxin associated with drug sensitivity to cisplatin, mitomycin C, doxorubicin, and etoposide. Cancer Res 55: 4293-4296, 1995.

71. Turk D, Hall MD, Chu BF, Ludwig JA, Fales HM, Gottesman MM and Szakacs G: Identification of compounds selectively killing multidrug-resistant cancer cells. Cancer Res 69: 8293-8301, 2009.

72. Van Jaarsveld MT, Helleman J, Berns EM and Wiemer EA MicroRNAs in ovarian cancer biology and therapy resistance. Int J Biochem Cell Biol 42: 1282-1290, 2010.

73. Sorrentino A, Liu CG, Addario A, Peschle C, Scambia G and Ferlini C: Role of microRNAs in drug-resistant ovarian cancer cells. Gynecol Oncol 111: 478-486, 2008.

74. Yang N, Kaur S, Volinia S, et al: MicroRNA microarray identifies Let-7i as a novel biomarker and therapeutic target in human epithelial ovarian cancer. Cancer Res 68: 10307-10314, 2008.

75. Boren T, Xiong Y, Hakam A, et al: MicroRNAs and their target messenger RNAs associated with ovarian cancer response to chemotherapy. Gynecol Oncol 113: 249-255, 2009. 
76. Kumar S, Kumar A, Shah PP, Rai SN, Panguluri SK and Kakar SS: MicroRNA signature of cis-platin resistant vs. cisplatin sensitive ovarian cancer cell lines. J Ovarian Res 4: 17, 2011.

77. Hilliard TS, Gaisina IN, Muehlbauer AG, Gaisin AM, Gallier F and Burdette JE: Glycogen synthase kinase 3beta inhibitors induce apoptosis in ovarian cancer cells and inhibit in-vivo tumor growth. Anticancer Drugs 22: 978-985, 2011.

78. Su HY, Lai HC, Lin YW, et al: Epigenetic silencing of SFRP5 is related to malignant phenotype and chemoresistance of ovarian cancer through Wnt signaling pathway. Int J Cancer 127: $555-567,2010$.

79. Bellone S, Siegel ER, Cocco E, et al: Overexpression of epithelial cell adhesion molecule in primary, metastatic, and recurrent/chemotherapy-resistant epithelial ovarian cancer: implications for epithelial cell adhesion molecule-specific immunotherapy. Int J Gynecol Cancer 19: 860-866, 2009.

80. Chen JY, Shen C, Yan Z, Brown DP and Wang M: A systems biology case study of ovarian cancer drug resistance. Comput Syst Bioinformatics Conf: pp389-398, 2006.

81. Shah MA and Schwartz GK: Cell cycle-mediated drug resistance: an emerging concept in cancer therapy. Clin Cancer Res 7: 2168-2181, 2001.

82. Miller DH, Fischer AK, Chu KF, Burr R, Hillenmeyer S Brard L and Brodsky AS: T0901317 inhibits cisplatin-induced apoptosis in ovarian cancer cells [corrected]. Int $\mathbf{J}$ Gynecol Cancer 21: 1350-1356, 2011.

83. Trinh XB, van Dam PA, Dirix LY, Vermeulen PB and Tjalma WA: The rationale for mTOR inhibition in epithelial ovarian cancer. Expert Opin Investig Drugs 18: 1885-1891, 2009.

84. Li J, Feng Q, Kim JM, et al: Human ovarian cancer and cisplatin resistance: possible role of inhibitor of apoptosis proteins. Endocrinology 142: 370-380, 2001.

85. Luo T, Yu J, Nguyen J, et al: Electron transfer-based combination therapy of cisplatin with tetramethyl-p-phenylenediamine for ovarian, cervical, and lung cancers. Proc Natl Acad Sci USA 109: 10175-10180, 2012.

86. Nessa MU, Beale P, Chan C, Yu JQ and Huq F: Combinations of resveratrol, cisplatin and oxaliplatin applied to human ovarian cancer cells. Anticancer Res 32: 53-59, 2012.

87. Kumaran GC, Jayson GC and Clamp AR: Antiangiogenic drugs in ovarian cancer. Br J Cancer 100: 1-7, 2009.

88. Zhang Y, Cheng Y, Ren X, et al: NAC1 modulates sensitivity of ovarian cancer cells to cisplatin by altering the HMGB1-mediated autophagic response. Oncogene 31: 1055-1064, 2012.

89. Buys TP, Chari R, Lee EH, et al: Genetic changes in the evolution of multidrug resistance for cultured human ovarian cancer cells. Genes Chromosomes Cancer 46: 1069-1079, 2007.

90. Auner V, Sehouli J, Oskay-Oezcelik G, Horvat R, Speiser P and Zeillinger R: ABC transporter gene expression in benign and malignant ovarian tissue. Gynecol Oncol 117: 198-201, 2010.

91. Chikazawa N, Tanaka H, Tasaka T, Nakamura M, Tanaka M, Onishi $\mathrm{H}$ and Katano M: Inhibition of Wnt signaling pathway decreases chemotherapy-resistant side-population colon cancer cells. Anticancer Res 30: 2041-2048, 2010.
92. Wang S, Li W, Xue Z, et al: Molecular imaging of p53 signal pathway in lung cancer cell cycle arrest induced by cisplatin. Mol Carcinog: Jun 5, 2012 (Epub ahead of print). doi: $10.1002 / \mathrm{mc} .21930$.

93. Leon-Galicia I, Diaz-Chavez J, Garcia-Villa E, et al: Resveratrol induces downregulation of DNA repair genes in MCF-7 human breast cancer cells. Eur J Cancer Prev 22: 11-20, 2013.

94. Han W, Pan H, Chen Y, et al: EGFR tyrosine kinase inhibitors activate autophagy as a cytoprotective response in human lung cancer cells. PLoS One 6: e18691, 2011.

95. Liu LZ, Zhou XD, Qian G, Shi X, Fang J and Jiang BH: AKT1 amplification regulates cisplatin resistance in human lung cancer cells through the mammalian target of rapamycin/ p70S6K1 pathway. Cancer Res 67: 6325-6332, 2007.

96. Chung AS, Kowanetz M, Wu X, et al: Differential drug classspecific metastatic effects following treatment with a panel of angiogenesis inhibitors. J Pathol 227: 404-416, 2012.

97. Waldner MJ and Neurath MF: Targeting the VEGF signaling pathway in cancer therapy. Expert Opin Ther Targets 16: 5-13, 2012.

98. Ishdorj G, Li L and Gibson SB: Regulation of autophagy in hematological malignancies: role of reactive oxygen species. Leuk Lymphoma 53: 26-33, 2012.

99. Liu L, Yang M, Kang R, et al: DAMP-mediated autophagy contributes to drug resistance. Autophagy 7: 112-114, 2011.

100. Fisher C, Coleman T and Plant N: Probabilistic orthology analysis of the ATP-binding cassette transporters: implications for the development of multiple drug resistance phenotype. Drug Metab Dispos 40: 1397-1402, 2012.

101. Shukla S, Chen ZS and Ambudkar SV: Tyrosine kinase inhibitors as modulators of $\mathrm{ABC}$ transporter-mediated drug resistance. Drug Resist Updat 15: 70-80, 2012.

102. Longley DB and Johnston PG: Molecular mechanisms of drug resistance. J Pathol 205: 275-292, 2005.

103. Johnson SW, Ozols RF and Hamilton TC: Mechanisms of drug resistance in ovarian cancer. Cancer 71: 644-649, 1993.

104. Sharan R, Ulitsky I and Shamir R: Network-based prediction of protein function. Mol Syst Biol 3: 88, 2007.

105. Behm-Ansmant I, Rehwinkel J and Izaurralde E: MicroRNAs silence gene expression by repressing protein expression and/ or by promoting mRNA decay. Cold Spring Harb Symp Quant Biol 71: 523-530, 2006.

106. Bartel DP: MicroRNAs: genomics, biogenesis, mechanism, and function. Cell 116: 281-297, 2004.

107. Kloosterman WP and Plasterk RH: The diverse functions of microRNAs in animal development and disease. Dev Cell 11: 441-450, 2006.

108. Croce CM and Calin GA: miRNAs, cancer, and stem cell division. Cell 122: 6-7, 2005

109. Tili E, Michaille JJ, Gandhi V, Plunkett W, Sampath D and Calin GA: miRNAs and their potential for use against cancer and other diseases. Future Oncol 3: 521-537, 2007. 